\title{
Radiation disease of the urinary tract: Histological features of 18 cases
}

\author{
U R Suresh, V J Smith, E W Lupton, N Y Haboubi
}

\begin{abstract}
Aims: To study the spectrum of histological changes in the urinary tract caused by pelvic irradiation.

Methods: Biopsy specimens of ureter and bladder and cystectomy specimens from 18 patients who had received pelvic irradiation were studied and the histological features were arbitrarily grouped into early and late changes.

Results: A wide range of histological changes were noted of which submucosal inflammation, fibrosis, and epithelial damage were the commonest. Other less common features were interstitial haemorrhage, ureteritis and cystitis cystica, squamous metaplasia of vaginal type and perineural inflammation.

Conclusions: Although epithelial changes were most prominent in early cases and stromal changes in the late cases, there was a continuing spectrum of epithelial damage which persisted many years after initial radiation. Similarly, submucosal fibrosis was equally prominent in early and late phases.
\end{abstract}

(F Clin Pathol 1993;46:228-231)

Injury to the urinary tract as a result of radiation is common but rarely biopsied. Experience of changes in early disease is limited, because of a lack of available biopsy specimens. In late disease, changes are mainly fibrovascular and epithelial damage has been thought to be minimal. ${ }^{2}$

The differences in radiosensitivity between the neoplasm and the adjacent normal tissue exposed to radiation causes a significant incidence of radiation injury to normal tissue. ${ }^{3}$ In the past 15-20 years there have been only occasional studies of the histological changes induced by irradiation of normal human urinary bladder. Antonakopoulos et al studied the ultrastructural changes in the human urinary bladder following irradiation and found that the microscopic changes, such as degeneration and extensive necrosis of bladder wall, were sufficient to explain the clinical sequelae of bladder irradiation. ${ }^{4}$

We studied material from 18 cases of radiation induced injury to ureters and urinary bladders and found evidence of continuing epithelial damage in the late phase. We have also documented the several histological changes observed in these biopsy specimens.

\section{Methods}

Fifteen biopsy specimens of urinary bladder and ureter and three cystectomy specimens from patients who had received radiotherapy to the pelvic organs were retrieved from the files at the University Hospital of South Manchester Pathology Department (Withington Hospital). Sixteen of these patients had been treated for carcinoma of the cervix and two for carcinoma of the body of uterus. The age range was 33 to 68 years. The patients had received between 3500 and 7500 cGys of caesium and external beam irradiation. The time interval between radiation and biopsy varied from six months to four years. Most patients presented with frequency, dysuria, incontinence and haematuria. There was no other coexisting cause of bladder disease, such as urinary tract infection.

The histological features we studied were documented in three groups:

Surface epithelium: ulceration, atypia, proliferation, vacuolation and squamous metaplasia.

Stroma/submucosa: oedema, fibrosis, atypical cells, vascular changes and presence of deep epithelial inclusions (ureteritis or cystitis cystica).

Inflammatory infiltrate: type of cells, location, and density.

We compared the differences between biopsy specimens taken within 12 months of radiation (early stage) and those taken after 12 months (late stage).

\section{Results}

The single most consistent finding (in all 18 patients) was an inflammatory cell infiltrate in the submucosa. Of these, most (12 of 18) had a mixed cell population with acute and chronic inflammatory cells. The remaining six contained polymorphs or lymphoplasmacytic cells. In a few cases the infiltrate was particularly prominent in the perineural regions, although we could find no obvious explanation for this (fig 1).

The second most common finding was submucosal fibrosis, which was seen in 15 of 18 cases and was present in both the early and late stage biopsy specimens. The fibrosis was histologically similar in both groups and had a glassy hyalinised appearance.

Surface ulceration was, however, seen more frequently in early specimens and was noted in five of six cases as opposed to only five of 12 of late specimens. Similarly, epithelial cell atypia (nuclear pleomorphism, hyperchromatism, and granular cytoplasm) was

\author{
Correspondence to: \\ Dr N Y Haboubi \\ Accepted for publication \\ 26 August 1992 \\ Histopathology \\ South Manchester. \\ Manchester \\ Department of \\ Urology
}




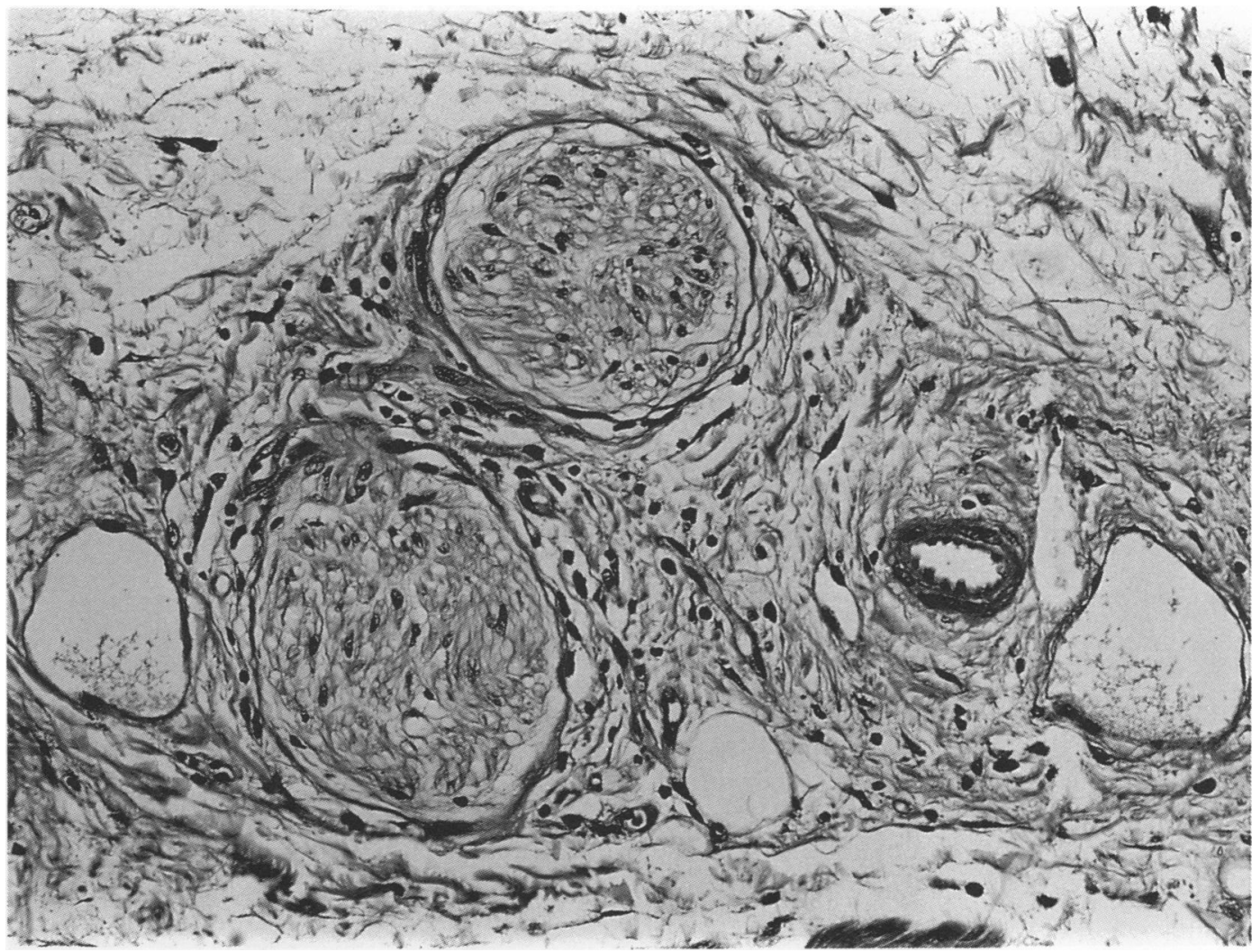

Figure 1 Perineural inflammation.

also found more frequently in early biopsy (two of six) than late biopsy specimens (two of twelve).

Cytoplasmic vacuolation and proliferation of epithelial cells was seen much more frequently in "late" biopsy specimens (seven of twelve) and was absent in all the "early" biopsy specimens.

Vascular changes such as luminal occlusion, ectasia, fibrinoid necrosis and hyalinisation of vessel walls was present in one of six of the early and in only one of 12 of the late biopsy specimens (fig 2).

In three of our cases two "late" and one "early") there were epithelial "inclusions" within the muscularis mucosa which were composed of well formed, dilated epithelial glands surrounded by a muscular sheath (fig 3 ). In two cases these were found in the ureteric wall and in one case, in the bladder wall.

In two of the 12 late cases there were haemosiderin deposits in the submucosa.

In one of the 12 late cases there was diffuse squamous metaplasia of vaginal type (fig 4).

\section{Discussion}

Injury induced by medication has been described in various organs. ${ }^{5}$ With the increasing use of radiotherapy for tumours, we have become increasingly aware of the complications. ${ }^{1}$ Cells which divide more rapidly, for example epithelial cells, are more susceptible to radiation damage and this is because the peak radiosensitivity of cells is at points $M$ and $\mathrm{G} 2$ of the cell cycle. Conversely, cells with a lower rate of turnover like endothelial cells and fibroblasts will show evidence of damage later. ${ }^{1}$

Pelvic radiation is a common procedure for cervical and endometrial carcinomas but pathologists' experience is mostly confined to the late manifestation of such damage. A lack of biopsy specimens in early disease has made it difficult to study the evolution of the disease.

Generally speaking, there are no diagnostic or pathognomonic markers of radiation induced damage. ${ }^{6}$ It is a combination of histological features that suggest radiation injury.

In this study we had six cases in which the biopsy specimens were taken within one year of receiving pelvic irradiation and 12 cases where biopsies had been done after one year of receiving radiation.

The cases of less than 12 months after radiation showed features of "early" changes: predominantly epithelial ulceration and epithelial cell atypia. However, submucosal fibrosis was seen in equal numbers in "early" and "late" cases. Inflammatory cells of a mixed variety were present in all cases. Contrary to what has been documented so far, submucosal fibrosis seems to be a change that occurs early in the disease and persists into the late stages. Inflammatory cells probably have an important role to play in causing the fibrosis of the early stages.

One other interesting observation was that epithelial cell damage, as shown by cytoplasmic vacuolation and cell proliferation, was present in late cases, suggesting that there is continuous damage to the epithelium even after the acute ulcers have healed. These 


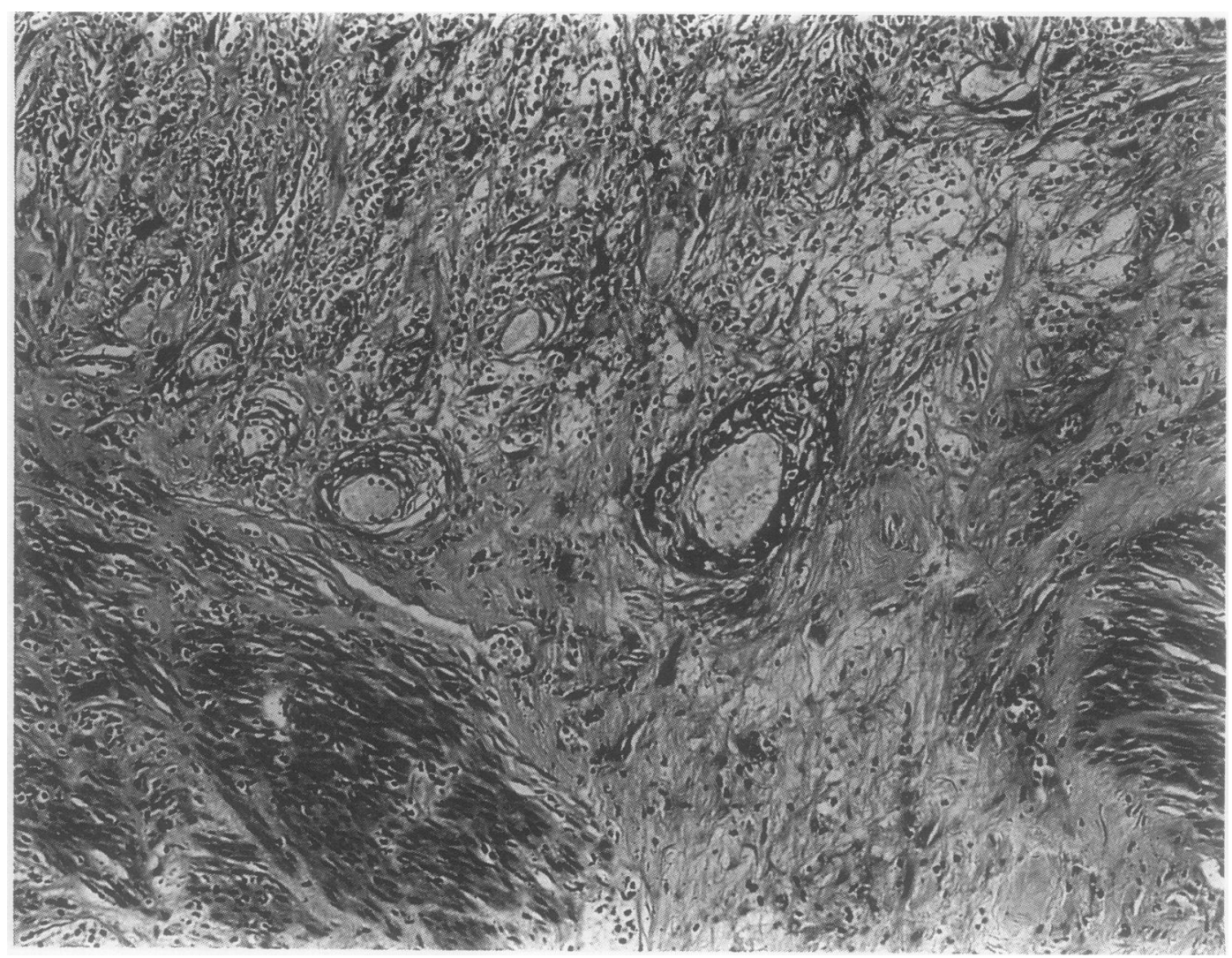

Figure 2 Fibrinoid necrosis of vessel walls.

changes were more profound in the bladder than in the ureters, presumably because the bladder acts as a reservoir for stagnant urine with resulting exposure to urinary constituents. The bladder is also exposed to larger doses of pelvic irradiation than the ureters.
The presence of cystic epithelial islands within the muscularis has not been described in association with irradiation. The exact mechanisms of ureteritis and cystitis cystica are unknown but the possible explanations are: (1) mucosal herniation due to raised

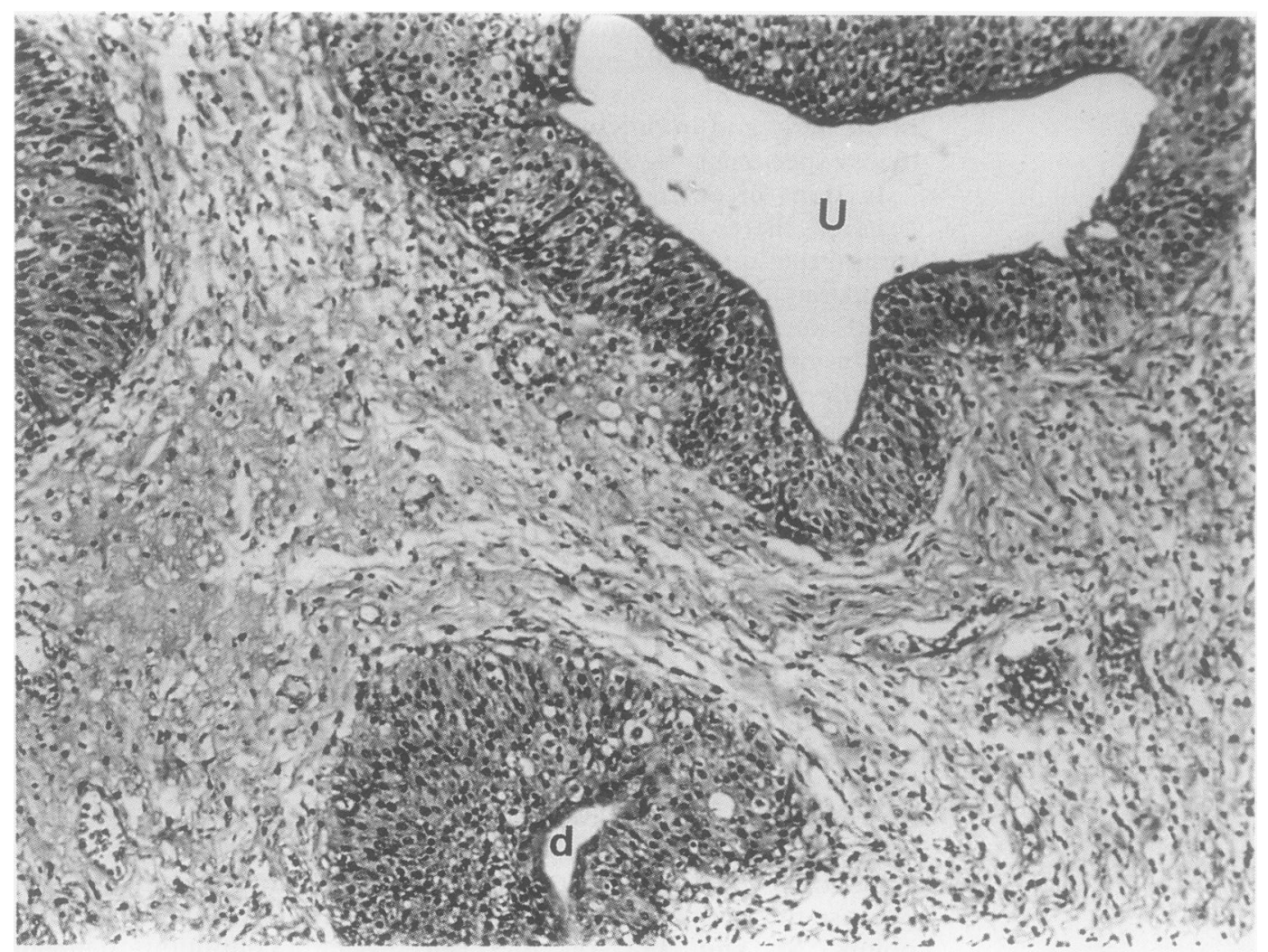

Figure 3 Ureteritis cystica (u: ureteric lumen, d: diverticulum in wall of ureter). 


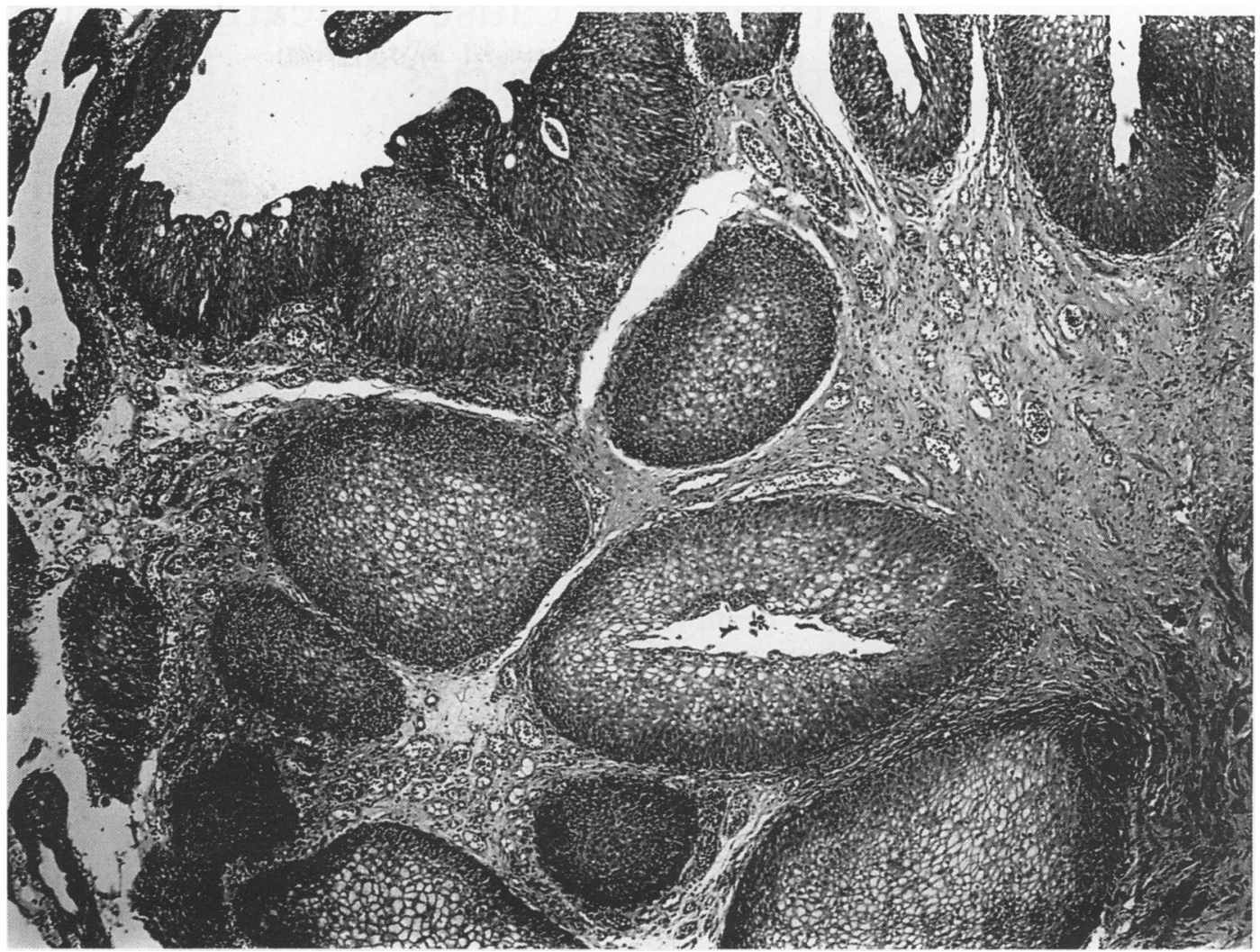

Figure 4 Squamous metaplasia of bladder epithelium.

intraluminal pressure; (2) mucosal herniation due to retractive fibrosis in submucosal region; (3) misplacement of glands during the regenerative process after ulceration.

The patients who had cystitis and ureteritis cystica and squamous metaplasia were in the younger age group (33, 40, and 43 years, respectively); we have no explanation for this. They had all received between 4000 and $4500 \mathrm{Cgys}$ of caesium and external beam radiation.

Unlike in the gastrointestinal tract, we did not notice a specific predilection for eosinophils in the inflammatory infiltrate. ${ }^{7}$ Another unusual finding in two of the late cases was patchy deposition of haemosiderin throughout the bladder wall. This was associated with vascular changes and is presumably the result of microhaemorrhages into the stroma. We found vascular changes in only one of the late biopsy specimens. It has been suggested that endothelial cells are responsible for vessel occlusion, with vascular spasm being an additional contributing factor. ${ }^{8}$ Thin-walled telangiectatic vessels, leading to massive haematuria, has been described following whole bladder irradiation. ${ }^{2}$

In summary, the most interesting observations were: the continuing spectrum of epithelial damage in late cases; the presence of "epithelial inclusions" within the muscular layer; and equal incidence of fibrosis in early and late cases.

We thank Miss M Garner for her secretarial assistance.

1 Haboubi NY, Hasleton PS. Pathology of radiation injury. In: Schofield PF, Lupton EW, eds. Pelvic radiation disIn: Schoriel Berlin: Springen Verlag, 1989:17-35.

2 Strickland P. Complications of radiotherapy. Br $\mathcal{f}$ Hosp Med 1980;23:552-65.

3 Berthrong. Pathological changes secondary to radiation. World f Surg 1986;10:155-70.

4 Antonakopoulos GN, Hicks RM, Berry RJ. The subcellular basis of damage to the human urinary bladder induced by irradiation. F Pathol 1984;143:103-16.

5 Cotran RS, Kumar VS, Robbins S. Environmental pathology - radiation injury. In: Robbin's pathologic basis of diseases. 4th Edn. 1989:506.

6 Fajardo LF. The pathology of radiation injury. Masson Publishing USA, Inc, 1982:6.

7 Haboubi NY, Schofield PF, Rowland P. The light and electron microscopic features of early and late phase radiation-induced proctitis. Am $\mathcal{F}$ Gastroenterol 1988;3: $1140-4$.

8 Hopwell JW. The late vascular effect of radiation. $\mathrm{Br} \mathcal{F}$ Radiol 1974;47:157-8. 